\title{
FORMAÇÃO DE AZEÓTROPOS DO PONTO DE VISTA DA TERMODINÂMICA DE SOLVATAÇÃo
}

\author{
Josefredo R. Pliego Jr.*,(i) \\ Departamento de Ciências Naturais, Universidade Federal de São João del-Rei, 36301-160 São João del-Rei - MG, Brasil
}

Recebido em 09/07/2021; aceito em 27/08/2021; publicado na web em 22/09/2021

\begin{abstract}
FORMATION OF AZEOTROPES FROM THE STANDPOINT OF SOLVATION THERMODYNAMICS. The formation of azeotropes is related to the deviation of the ideality of liquid mixtures. This deviation is expressed by the activity coefficient of each component. However, the physical origin of this behavior is not well quantified in terms of molecular interactions. In this work, a regular solution model was used, relating the $\beta$ parameter to the solvation free energy of pure liquids in themselves and in the other component. A mathematical expression was derived that indicates when an azeotrope is formed. This equation shows that the greater the difference in vapour pressure between components, the greater the solvation difference of each liquid in itself and in the other component is needed to form the azeotrope. A set of 10 mixtures were randomly chosen and investigated as a test, and the equation worked in all cases.
\end{abstract}

Keywords: Margules equation; liquid-vapour equilibrium; Redlich-Kister expansion; infinity dilution activity coefficient.

\section{INTRODUÇÃO}

Equilíbrio de fases em misturas é um tópico clássico de físicoquímica e discutido em todos os livros-texto da área. ${ }^{1-4} \mathrm{O}$ ponto de partida para discussão desse tópico é a termodinâmica de soluções. Em sistemas com fases líquida e gasosa, o equilíbrio termodinâmico é baseado nas leis de Raoult e de Henry. A primeira se aplica a situação em que o componente de interesse está altamente concentrado, com sua fração molar perto de 1 . Já a lei de Henry se aplica quando o componente de interesse está diluído, com sua fração molar perto de zero. A lei de Raoult é especialmente interessante como ponto de partida para discussão de equilíbrio de fases. Quando a mesma é válida sobre todo intervalo de composição, diz-se que a solução é ideal. Soluções ideais são favoráveis a formação de misturas. Do ponto de vista microscópico, a solução ideal seria aquela em que as interações moleculares entre moléculas diferentes seriam semelhantes, assim como os tamanhos moleculares.

Usualmente, as soluções não seguem a idealidade e desvios da lei de Raoult são comuns, podendo ser pequenos ou muito acentuados. Grandes desvios da idealidade levam a efeitos muito interessantes, como separação de duas fases líquidas, e a formação de azeótropos no caso de equilíbrio líquido-vapor. A forma de expressar o desvio da idealidade é através do coeficiente de atividade, $\gamma_{i}\left(T, x_{i}\right)$, que é principalmente uma função da temperatura $\mathrm{T}$ e da composição, representada aqui pela fração molar do componente i, $x_{i}$ (discussão restrita a soluções binárias). Várias equações para expressar a energia de Gibbs de mistura, e como $\gamma_{i}$ varia com a composição, foram propostas, como a equação de Margules, ${ }^{5,6}$ expansão de RedlichKister, ${ }^{7}$ equação de Wilson ${ }^{8}$ e modelo NRTL $^{9}$ para citar os mais conhecidos. Apesar da indiscutível utilidade dessas equações, o coeficiente de atividade carece de um significado físico mais claro e facilmente conectado a interação intermolecular. Considerando que um dos aspectos importantes da físico-química é o physical insights, é desejável entendermos de forma clara, em termos de interações entre moléculas, qual a origem física de eventos como separação de fases e formação de azeótropos.

Nos últimos 30 anos, o conceito de energia livre de solvatação $0^{10-14}$ tem se estabelecido firmemente na comunidade de físico-químicos

*e-mail: pliego@ufsj.edu.br
(Figura 1), sendo largamente utilizado pelos químicos teóricos e computacionais para descrever diferentes processos em fase líquida, como equilíbrio químico, ${ }^{15,16}$ cinética de reação, ${ }^{17-20}$ efeito no espectro de absorção, ${ }^{21,22}$ equilíbrio de fases, ${ }^{23-26}$ etc. Com a crescente utilização de métodos computacionais na química, com impacto nas diferentes áreas, o conceito também vai se disseminando nos demais domínios da química. Mesmo a área de engenharia química já vem incorporando esse conceito. ${ }^{27}$

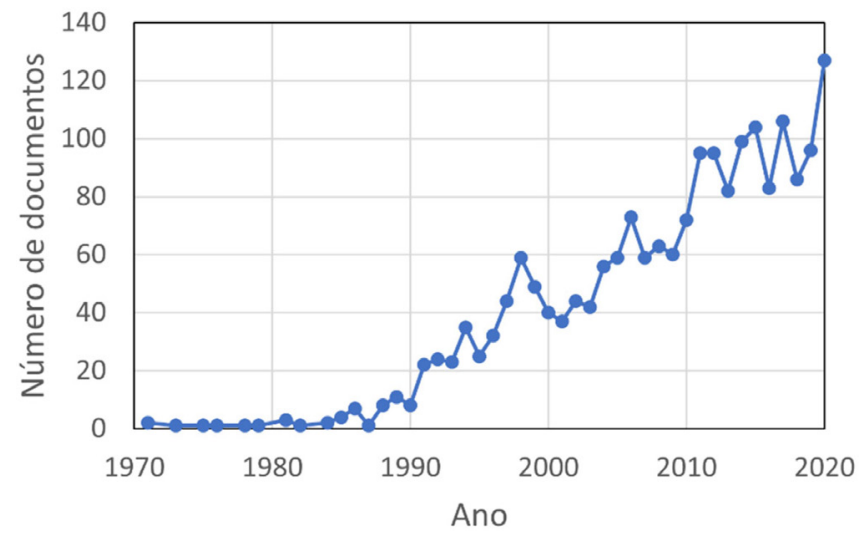

Figura 1. Pesquisa pelo termo "solvation free energy" na base Scopus

Recentemente, mostrou-se que o processo de separação de fases em sistemas binários pode ser predito por um modelo de solução regular ${ }^{5}$ utilizando um parâmetro $\beta,{ }^{26}$ dado por:

$\beta=\frac{1}{2}\left(\Delta G_{\text {solv }}^{*}(1: 2)+\Delta G_{\text {solv }}^{*}(2: 1)-\Delta G_{\text {solv }}^{*}(1: 1)-\Delta G_{\text {solv }}^{*}(2: 2)\right)$

em que o conceito de energia livre de solvatação é inserido. Nessa equação, $\Delta G_{\text {solv }}^{*}(1: 2)$ e $\Delta G_{\text {solv }}^{*}(2: 1)$ são as energias livres do componente 1 em 2 e de 2 em 1 , em diluição infinita. Os valores de $\Delta G_{\text {solv }}^{*}(1: 1)$ e $\Delta G_{\text {solv }}^{*}(2: 2)$ são as energias livres de solvatação de cada componente nele mesmo. Como é bem estabelecido na termodinâmica de soluções líquidas binárias, ${ }^{5}$ a separação de fases ocorre quando a derivada segunda a energia de Gibbs de mistura com relação a fração molar de um dos componentes fica negativa em algum ponto. No caso de solução regular, essa condição é satisfeita quando: ${ }^{5,26}$ 


$$
\beta>2 R T
$$

Fisicamente, isso significa que cada componente terá de ser, na média, mais solvatado na sua fase pura por 2RT em relação a sua solvatação no outro componente. Dessa forma, temos uma visão física clara do fenômeno de separação de fases em termos de interação molecular e do conceito moderno de energia livre de solvatação.

No presente manuscrito, o objetivo é estender essa análise do efeito da energia livre de solvatação sobre o comportamento de equilíbrio de fases. Iremos analisar o processo de formação de azeótropos usando novamente o modelo de solução regular, combinado com o conceito de energia livre de solvatação. Para verificar a performance dessa abordagem, foi utilizado um modelo mais completo para calcular o coeficiente de atividade, a equação de Margules de dois parâmetros, que pode ser derivada da expansão de Redlich-Kister da energia livre de mistura. ${ }^{5,7}$ Os parâmetros desse modelo foram obtidos de dados experimentais de coeficiente de atividade em diluição infinita disponíveis na literatura. Um total de 10 soluções binárias diversas foram investigadas. Deve-se mencionar que o modelo de solução regular tem sido utilizado para discussão geral do equilíbrio de fases. ${ }^{28-30}$

\section{METODOLOGIA}

\section{Modelo de solução regular}

A pressão de vapor de um componente i em uma mistura líquida com fração molar $x_{i}$ é dado por:

$$
p_{i}=\gamma_{i} x_{i} p_{i}^{o}
$$

sendo $p_{i}^{o}$ a pressão de vapor do líquido puro e $\gamma_{i}$ o coeficiente de atividade. Usando a equação de Margules de um parâmetro, ${ }^{6}$ a variação de $\gamma_{i}$ com a composição é dada por:

$$
\gamma_{1}=e^{\beta x_{2}^{2} R T}
$$

$$
\gamma_{2}=e^{\beta x_{1}^{2} / R T}
$$

sendo $\beta$ um parâmetro do modelo, que pode ser associado ao coeficiente de atividade em diluição infinita $\left(\gamma_{i}^{\infty}\right)$. De fato, fazendo $x_{2} \rightarrow 1$ em 4 e $x_{1} \rightarrow 1$ em 5, fica:

$$
\gamma_{1}^{\infty}=\gamma_{2}^{\infty}=e^{\beta / R T}
$$

Na realidade, os valores de $\gamma_{i}^{\infty}$ não são usualmente iguais. Sendo assim, para termos uma forma funcional para $\gamma_{i}$ dada por (4) e (5), podemos fazer uma média para obter $\beta$, ou seja:

$$
\beta=\frac{1}{2}\left(R T \ln \gamma_{1}^{\infty}+R T \ln \gamma_{2}^{\infty}\right)=R \operatorname{Tln}\left(\gamma_{m}^{\infty}\right)
$$

sendo o coeficiente de atividade em diluição infinita médio dado por:

$$
\gamma_{m}^{\infty}=\left(\gamma_{1}^{\infty} \gamma_{2}^{\infty}\right)^{1 / 2}
$$

Equações (7) e (8) foram utilizadas para obter $\beta$ e $\gamma_{m}{ }^{\infty}$.

A energia livre de solvatação, como definido por Ben-Naim, ${ }^{10,1}$ pode ser escrita como a energia livre de partição de um composto i entre as fases gasosa e solução líquida:

$$
\frac{[i]_{\text {sol }}}{[i]_{g}}=e^{-\Delta G_{\text {solv }} / R T}
$$

No caso de fase gasosa, podemos escrever:

$$
p_{i}=[i]_{g} \tilde{R} T
$$

sendo $\tilde{R}$ a constante dos gases em atm.L.K ${ }^{-1} \cdot \mathrm{mol}^{-1}$. Substituindo, fica:

$$
\Delta G_{s o l v}(i)=R T \ln \left(p_{i}\right)-R T \ln [i]_{s o l}-R T \ln (\tilde{R} T)
$$

No caso de i sendo solvatado em seu líquido puro, temos:

$$
\Delta G_{\text {solv }}(i: i)=R T \ln \left(p_{i}^{o}\right)-R T \ln \frac{\rho_{i}}{M_{i}}-R T \ln (\tilde{R} T)
$$

E no caso de i ser solvatado pelo solvente j, em diluição infinita, fica:

$\Delta G_{\text {solv }}(i: j)=R T \ln \left(\gamma_{i}^{\infty}\right)+R T \ln \left(p_{i}^{o}\right)-R T \ln \frac{\rho_{j}}{M_{j}}-R T \ln (\tilde{R} T)$

Combinando (12) e (13), teremos:

$$
R T \ln \left(\gamma_{i}^{\alpha}\right)=\Delta G_{\text {solv }}(i: j)-\Delta G_{\text {solv }}(i: i)+R T \ln \frac{\rho_{j} M_{i}}{M_{j} \rho_{i}}
$$

Considerando os componentes 1 e 2 , podemos escrever:

$$
\begin{gathered}
R T \ln \left(\gamma_{1}^{\infty}\right)=\Delta G_{\text {solv }}(1: 2)-\Delta G_{\text {solv }}(1: 1)+R T \ln \frac{\rho_{2} M_{1}}{M_{2} \rho_{1}} \\
R \operatorname{Tln}\left(\gamma_{2}^{\infty}\right)=\Delta G_{\text {solv }}(2: 1)-\Delta G_{\text {solv }}(2: 2)+R T \ln \frac{\rho_{1} M_{2}}{M_{1} \rho_{2}}
\end{gathered}
$$

Usando (15) e (16) em (7), teremos:

$\beta=\frac{1}{2}\left(\Delta G_{\text {solv }}^{*}(1: 2)+\Delta G_{\text {solv }}^{*}(2: 1)-\Delta G_{\text {solv }}^{*}(1: 1)-\Delta G_{\text {solv }}^{*}(2: 2)\right)$

A equação (17) é a mesma utilizada no estudo do equilíbrio de fases líquido-líquido, ${ }^{26}$ mas no presente estudo não se fez nenhum pressuposto sobre como $\Delta \mathrm{G}_{\text {solv }}$ se comportaria com a composição da mistura. Usou-se a forma funcional da equação de Margules de um parâmetro para $\gamma_{i}$, as equações (4) e (5), e o valor médio de $\gamma_{i}^{\infty}$, dado pela equação (7). As equações (15) e (16) são exatas. Observando as equações (4), (5) e (17), notamos a clara conexão com o modelo de solução regular em que $\beta$ estaria associado a uma entalpia de mistura. ${ }^{5}$ No presente modelo, $\beta$ está relacionado a diferença entre a energia livre de solvatação em diluição infinita e no líquido puro dado por (17).

\section{Formação de azeótropo}

A formação de azeótropo a uma certa temperatura ocorre quando a composição das fases líquida e gasosa são iguais, resultando que: ${ }^{6}$

$$
\gamma_{1} p_{1}^{o}=\gamma_{2} p_{2}^{o}
$$

Usando a forma funcional de $\gamma_{i}$, temos:

$$
e^{\beta x_{2}^{2} / R T} p_{1}^{o}=e^{\beta x_{1}^{2} / R T} p_{2}^{o}
$$

Tomando os logaritmos e rearranjando, fica:

$$
x_{2}^{2}-x_{1}^{2}=\frac{R T}{\beta} \ln \left(\frac{p_{2}^{o}}{p_{1}^{o}}\right)
$$

$$
x_{1}=\frac{1}{2}\left(1-\frac{R T}{\beta} \ln \left(\frac{p_{2}^{o}}{p_{1}^{o}}\right)\right)
$$


Essa equação mostra a composição do azeótropo. Isso significa que a fração molar $x_{1}$ deve estar entre 0 e 1, ou seja, para formar azeótropo, a condição é:

$$
0<1-\frac{R T}{\beta} \ln \left(\frac{p_{2}^{o}}{p_{1}^{o}}\right)<2
$$

Essa equação leva a duas condições para formar azeótropo, o de pressão total máxima $(\beta>0)$ e o de pressão total mínima $(\beta<0)$, a seguir:

$$
\begin{gathered}
\beta>R T\left|\ln \left(\frac{p_{2}^{o}}{p_{1}^{o}}\right)\right| \\
\beta<-R T\left|\ln \left(\frac{p_{2}^{o}}{p_{1}^{o}}\right)\right|
\end{gathered}
$$

Essas duas equações foram utilizadas para prever a formação de azeótropo neste estudo. Como pode-se notar, a equação (23) mostra que a formação de azeótropo está relacionada a razão de pressão de vapor entre os componentes e a diferença de energia livre de solvatação, medida por $\beta$. Quanto maior a razão da pressão de vapor, menos solvatado deve estar o componente 1 na solução de 2 e reciprocamente para $2 \mathrm{em} 1$. No caso de (24), é o contrário, uma maior razão de pressão de vapor dos componentes puros implica que o componente 1 deve estar mais solvatado em 2 do que em 1 , e similarmente para a solvatação de 2 em 1 .

\section{Equação de Margules de dois parâmetros}

Para uma análise mais acurada do equilíbrio líquido-vapor, e poder avaliar graficamente a previsão das equações (23) e (24), foi utilizada a equação de Margules com dois parâmetros para os coeficientes de atividade. ${ }^{6}$ Essa equação está relacionada com a expansão de Redlich-Kister da energia livre de mistura não ideal, ${ }^{5-7}$ e preferiu-se utilizar a forma da função relacionada a essa expansão. Nessa abordagem, os coeficientes de atividade são dados por:

$$
\gamma_{1}=e^{\left((\mathcal{B}+3 \mathcal{C}) x_{2}^{2}-4 \mathcal{C} x_{2}^{3}\right) / R T}
$$

$\mathrm{e}$

$$
\gamma_{2}=e^{\left((\mathcal{B}-3 \mathcal{C}) x_{1}^{2}+4 \mathcal{C} x_{1}^{3}\right) / R T}
$$

Os parâmetros $\mathcal{B}$ e $\mathcal{C}$ são obtidos de pelas relações:

$$
\begin{aligned}
& \mathcal{B}=\frac{1}{2}\left(R \operatorname{Tln} \gamma_{1}^{\infty}+R T \ln \gamma_{2}^{\infty}\right) \\
& \mathcal{C}=\frac{1}{2}\left(R T \ln \gamma_{2}^{\infty}-R T \ln \gamma_{1}^{\infty}\right)
\end{aligned}
$$

Observe que $\beta$ da solução regular e $\mathcal{B}$ da expansão tem a mesma relação com $\gamma_{i}^{\infty}$. Foi considerado que $\mathcal{B}$ e $\mathcal{C}$ são independentes da temperatura. A pressão total da mistura é obtida de:

$$
P=x_{1} \gamma_{1} p_{1}^{o}+x_{2} \gamma_{2} p_{2}^{o}
$$

\section{Dados de $p_{i}^{\infty} \mathbf{e} \gamma_{i}^{\infty}$}

Para a análise de formação de azeótropos, foram utilizados dados de pressão de vapor dos componentes puros $\left(p_{i}^{o}\right)$ obtidos do NIST ${ }^{31}$ pela equação de Antoine, e dados de $\gamma_{i}^{\infty}$ de 10 misturas obtidos da literatura. ${ }^{25,32,33}$ A tabela completa dos dados está em material suplementar.

\section{RESULTADOS E DISCUSSÃO}

As misturas binárias investigadas neste trabalho têm valores tão altos de $\beta$ quanto $5 \mathrm{~kJ} \mathrm{~mol}^{-1}$, mostrando um substancial desvio da idealidade, quanto valores tão baixos como $0,6 \mathrm{~kJ} \mathrm{~mol}^{-1}$, sendo uma solução com pouco desvio da idealidade. Desvios excessivos a ponto de separar duas fases líquidas não foram considerados. Os resultados da análise de formação de azeótropos, segundo os critérios das equações (23) ou (24), estão mostrados na Tabela 1. As misturas que formam azeótropos com seus diagramas $\mathrm{P}$ versus $\mathrm{X}_{1}$ estão mostrados na Figura 2.

A primeira mistura é clorofórmio-metanol, que tem um alto desvio da idealidade $\left(\beta=4,06 \mathrm{~kJ} \mathrm{~mol}^{-1}\right)$ e forma um azeótropo em $\mathrm{X}_{1}=0,64$ a $330 \mathrm{~K}$, conforme mostra o gráfico e de acordo com a equação (23). Do ponto de vista de $\Delta \mathrm{G}_{\text {solv }}$, os dados mostram que é a variação da energia livre de solvatação do metanol indo do líquido puro à solução de clorofórmio que gera o maior desvio da idealidade. Já na segunda mistura, clorofórmio-acetonitrila, a variação de $\Delta \mathrm{G}_{\text {solv }}$ é pequena para ambos os solutos, resultando num pequeno $\beta$ de $0.6 \mathrm{~kJ} \mathrm{~mol}^{-1}$ e não havendo formação de azeótropo, de acordo com equação (23). Na terceira mistura, clorofórmio-tetraidrofurano, ambos os componentes são mais solvatados no outro componente do que a correspondente auto-solvatação, resultando em um $\beta$ negativo por $-3,54 \mathrm{~kJ} \mathrm{~mol}^{-1}$ e satisfazendo (24). Como consequência, há formação de um azeótropo negativo conforme mostra a Figura 2.

A quarta mistura é água-metanol, que tem um modesto desvio da idealidade e não forma azeótropo. A quinta mistura, água-piridina, tem um forte desvio da idealidade e satisfaz facilmente a equação (23), formando um azeótropo. Ambos os componentes são mais solvatados nos seus líquidos puros do que no outro componente. Já a sexta mistura, nitrometano-benzeno, também tem um substancial desvio da idealidade, mas o termo $\left|\mathrm{RT} \ln \left(\mathrm{p}_{2} / \mathrm{p}_{1}\right)\right|$ é substancial e próximo de $\beta$, de forma que o azeótropo é menos bem caracterizado do que os demais. Ambos os componentes contribuem para o valor de $\beta$ devido a variação de $\Delta \mathrm{G}_{\text {solv }}$. Nas duas misturas subsequentes, dietilaminaclorobenzeno e dietilamina-hexano, ambas têm um valor pequeno de $\beta$, sendo que os componentes apresentam solvatação muito similares no líquido puro e em diluição infinita no outro componente. Como resultado, não formam azeótropos.

A nona mistura, etanol-éter etílico, tem um desvio substancial da idealidade, $\operatorname{com} \beta=2,70 \mathrm{~kJ} \mathrm{~mol}^{-1}$, mas não forma azeótropo pela equação (23) a $310 \mathrm{~K}$ pelo fato de que $\left|\mathrm{RT} \ln \left(\mathrm{p}_{2} / \mathrm{p}_{1}\right)\right|$ é muito grande, o que é experimentalmente verificado, assim como é verificado pelo modelo usando a expansão de Redlich-Kister. A décima e última mistura, acetona-metanol, forma azeótropo como mostra a equação (23) e o gráfico na Figura 2. Nota-se que $\beta$ é levemente acima de $\left|\mathrm{RT} \ln \left(\mathrm{p}_{2} / \mathrm{p}_{1}\right)\right|$, e o gráfico é de um azeótropo pouco acentuado. Em termos de $\Delta \mathrm{G}_{\text {solv }}$, é o metanol que mais contribui para o desvio da idealidade ao ir de seu líquido puro para a solução de acetona.

Em resumo, todas as 10 misturas analisadas aqui pelas equações (23) e (24) estão de acordo com dados experimentais de formação de azeótropo, suportando a aplicação dessas relações e sua interpretação em termos de variação de energia livre de solvatação.

Recentemente, Roese e colaboradore ${ }^{24}$ propuseram uma relação simples para prever formação de azeótropos, usando energia livre de solvatação. Pelo critério daqueles autores, o azeótropo seria formado ao satisfazer simultaneamente as equações:

$$
\Delta G_{\text {solv }}^{*}(1: 2)>\Delta G_{\text {solv }}^{*}(1: 1) \wedge \Delta G_{\text {solv }}^{*}(2: 1)>\Delta G_{\text {solv }}^{*}(2: 2)
$$


Tabela 1. Análise de formação de azeótropos em soluções binárias ${ }^{a}$

\begin{tabular}{|c|c|c|c|c|c|c|c|c|}
\hline \multirow{2}{*}{ Comp. 1} & \multirow{2}{*}{ Comp. 2} & \multirow{2}{*}{$\beta^{\mathrm{b}}$} & \multirow{2}{*}{$\pm\left|\mathrm{RT} \ln \left(\mathrm{p}_{2} / \mathrm{p}_{1}\right)\right|^{\mathrm{b}}$} & \multirow{2}{*}{ Forma azeótropo? } & \multicolumn{4}{|c|}{$\Delta \mathrm{G}_{\text {solv }}{ }^{\mathrm{b}}$} \\
\hline & & & & & $(1: 2)$ & $(2: 1)$ & $(1: 1)$ & $(2: 2)$ \\
\hline clorofórmio & metanol & 4,06 & 1,05 & $\operatorname{sim}$ & $-17,0$ & $-12,7$ & $-17,5$ & $-20,3$ \\
\hline clorofórmio & acetonitrila & 0,60 & 1,95 & não & $-17,9$ & $-19,0$ & $-17,5$ & $-20,5$ \\
\hline clorofórmio & THF & $-3,54$ & $-0,45$ & $\operatorname{sim}$ & $-20,3$ & $-22,2$ & $-17,5$ & $-17,9$ \\
\hline $\mathrm{H}_{2} \mathrm{O}$ & metanol & 0,92 & 4,16 & não & $-23,6$ & $-21,3$ & $-26,4$ & $-20,3$ \\
\hline $\mathrm{H}_{2} \mathrm{O}$ & piridina & 5,06 & 0,34 & sim & $-20,0$ & $-19,4$ & $-26,4$ & $-23,1$ \\
\hline nitrometano & benzeno & 3,07 & 2,57 & $\operatorname{sim}$ & $-18,6$ & $-16,9$ & $-22,7$ & $-18,9$ \\
\hline dietilamina & clorobenzeno & 0,65 & 7,27 & não & $-16,2$ & $-22,9$ & $-16,5$ & $-23,9$ \\
\hline dietilamina & hexano & 0,75 & 0,99 & não & $-15,0$ & $-16,9$ & $-16,5$ & $-16,9$ \\
\hline $\mathrm{EtOH}$ & $\mathrm{Et}_{2} \mathrm{O}$ & 2,70 & 5,31 & não & $-17,5$ & $-12,9$ & $-21,3$ & $-14,5$ \\
\hline acetona & metanol & 1,75 & 1,47 & $\operatorname{sim}$ & $-16,9$ & $-17,2$ & $-17,3$ & $-20,3$ \\
\hline
\end{tabular}

a - Análise a $25^{\circ} \mathrm{C} . \mathrm{b}$ - Unidades de $\mathrm{kJ} \mathrm{mol}^{-1}$.

ou
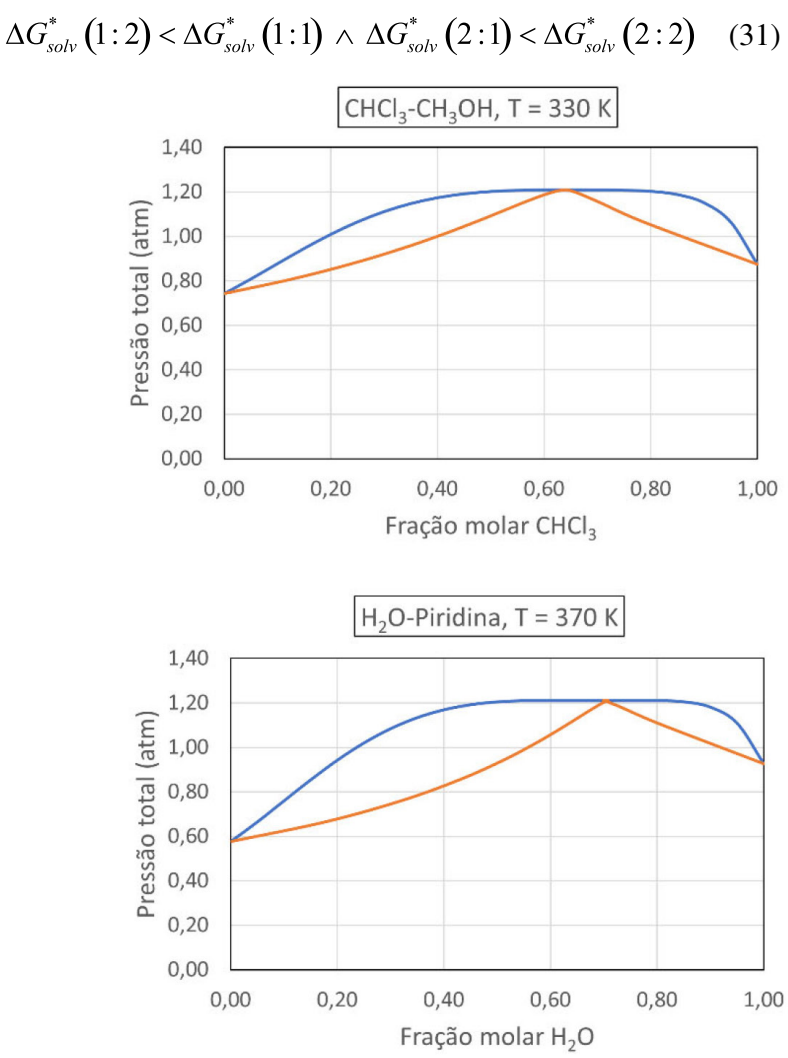

Observando os dados da Tabela 1, vemos que esses critérios falham nas misturas 7, 8 e 9. Dessa forma, os critérios estabelecidos neste artigo são mais acurados.
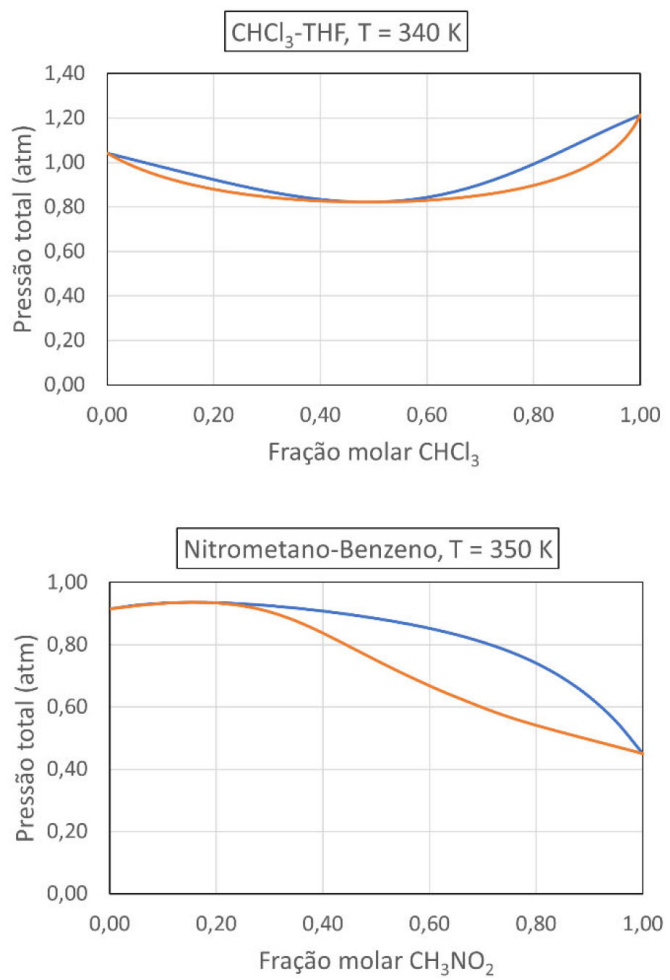

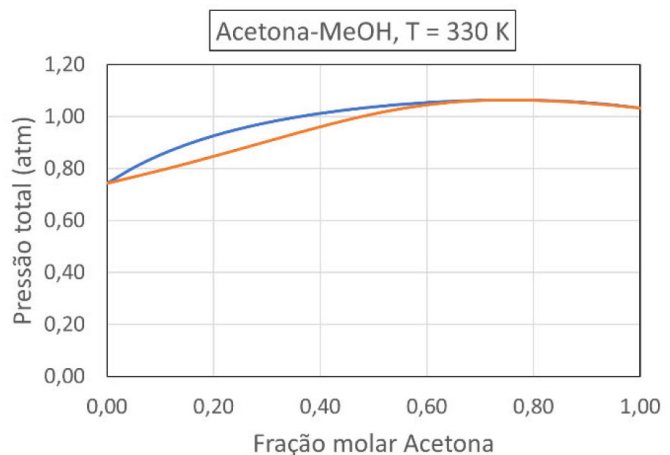

Figura 2. Diagramas de fases líquido-vapor das soluções que formam azeótropos obtido pelas equações 25 a 29 


\section{CONCLUSÕES}

O processo de formação de azeótropos foi analisado do ponto de vista da energia livre de solvatação dos componentes em si mesmos e no outro componente. Esses valores de energia livre de solvatação definem o parâmetro $\beta$. As equações (23) e (24) estabelecem os critérios para formação de azeótropos e foram testadas para 10 misturas binárias, funcionando em todos os casos. Essas equações mostram que a formação de azeótropos requerem que a variação de $\Delta \mathrm{G}_{\text {solv }}$ quando os componentes puros são transferidos para a solução do outro componente devem ser grandes o suficiente (positivo ou negativo) para superar a razão das pressões de vapor dos componentes puros. Colocando de outra forma, quanto maior a diferença de temperatura de ebulição entre os componentes, maior deve ser a diferença de energia livre de solvatação dos componentes imerso no outro componente em relação a sua própria solvatação para formar azeótropo.

\section{MATERIAL SUPLEMENTAR}

A tabela dos parâmetros utilizados pode ser acessada gratuitamente em http://quimicanova.sbq.org.br, em formato pdf.

\section{AGRADECIMENTOS}

O autor agradece ao CNPq pelo suporte.

\section{REFERÊNCIAS}

1. Engel, T.; Reid, P.; Physical Chemistry: Thermodynamics, Statistical Thermodynamics and Kinetics; $4^{\text {th }}$ ed., Pearson: London, 2019.

2. Levine, I. N. Physical Chemistry, $6^{\text {th }}$ ed., McGraw Hill: Singapore, 2009.

3. McQuarrie, D. A.; Simon, J. D.; Physical Chemistry: A Molecular Approach, University Science Books: Sausalito, 1997.

4. Castellan, G. W. Physical Chemistry; $3^{\text {rd }}$ ed.; Addison Wesley: Boston, 1983.

5. With, G. d.; Liquid-State Physical Chemistry: Fundamentals, Modeling and Applications, Wiley-VCH: Weinheim, 2013.

6. Prausnitz, J. M.; Lichtenthaler, R. N.; Azevedo, E. G.; Molecular Thermodynamics of Fluid-Phase Equilibria, $3^{\text {rd }}$ ed., Prentice Hall: Hoboken, 1999.
7. Redlich, O.; Kister, A. T.; Ind. Eng. Chem. 1948, 40, 345.

8. Wilson, G. M.; J. Am. Chem. Soc. 1964, 86, 127.

9. Renon, H.; Prausnitz, J. M.; AlChE J. 1968, 14, 135.

10. Ben-Naim, A.; Marcus, Y.; J. Chem. Phys. 1984, 81, 2016.

11. Ben-Naim, A.; J. Phys. Chem. 1978, 82, 792.

12. Pliego Jr, J. R.; Quim. Nova 2006, 29, 535.

13. Lin, Y.-L.; Aleksandrov, A.; Simonson, T.; Roux, B.; J. Chem. Theory Comput. 2014, 10, 2690.

14. Ben-Naim, A.; Molecular Theory of Solutions; Oxford University Press: New York, 2006.

15. Pliego Jr, J. R.; Riveros, J. M.; Wiley Interdiscip. Rev. Comput. Mol. Sci. 2020, $10, \mathrm{e} 1440$.

16. Marenich, A. V.; Ho, J.; Coote, M. L.; Cramer, C. J.; Truhlar, D. G.; Phys. Chem. Chem. Phys. 2014, 16, 15068.

17. Acevedo, O.; Jorgensen, W. L.; Wiley Interdiscip. Rev. Comput. Mol. Sci. 2014, $4,422$.

18. Dalessandro, E. V.; Pliego Jr, J. R.; Quim. Nova 2018, 41, 628.

19. Silva, N. M.; Deglmann, P.; Pliego, J. R.; J. Phys. Chem. B 2016, 120, 12660.

20. Miguel, E. L. M.; Santos, C. I. L.; Silva, C. M.; Pliego Jr, J. R.; J. Braz. Chem. Soc. 2016, 27, 2055.

21. Cramer, C. J.; Truhlar, D. G.; Chem. Rev. 1999, 99, 2161.

22. Marenich, A. V.; Cramer, C. J.; Truhlar, D. G.; Guido, C. A.; Mennucci, B.; Scalmani, G.; Frisch, M. J.; Chem. Sci. 2011, 2, 2143.

23. Klamt, A.; Eckert, F.; Fluid Phase Equilibr. 2000, 172, 43.

24. Roese, S. N.; Margulis, G. V.; Schmidt, A.; Uzat, C. B.; Heintz, J. D.; Paluch, A. S.; Ind. Eng. Chem. Res. 2019, 58, 22626.

25. Lisboa, F. M.; Pliego, J. R.; J. Mol. Model. 2018, 24, 56.

26. Pliego Jr, J. R.; J. Braz. Chem. Soc. 2015, 26, 1737.

27. Lin, S.-T.; Hsieh, C.-M.; Lee, M.-T.; J. Chin. Inst. Chem. Eng. 2007, 38, 467.

28. Novák, J. P.; Řehák, K.; Voňka, P.; Matouš, J.; Fluid Phase Equilibr. 2003, 208, 199.

29. Akkermans, R. L. C.; J. Phys. Chem. B 2017, 121, 1675.

30. Castellanos-Suárez, A. J.; García-Sucre, M.; Chem. Eng. Sci. 2012, 68, 443.

31. NIST Chemistry WebBook, NIST Standard Reference Database Number 69; National Institute of Standards and Technology, 2021.

32. Schreiber, L. B.; Eckert, C. A.; Ind. Eng. Chem. Process Des. Dev. 1971, 10,572 .

33. Coutinho, J. P.; Macedo, E. A.; Fluid Phase Equilibr. 1994, 95, 149. 